Full Length Article

\title{
Numerical investigation of oblique detonations induced by a finite wedge in a stoichiometric hydrogen-air mixture
}

\author{
Yishen Fang ${ }^{\mathrm{a}, \mathrm{b}, \mathrm{c}}$, Zongmin $\mathrm{Hu}^{\mathrm{b}, \mathrm{c}}$, Honghui Teng ${ }^{\mathrm{a}, *}$ \\ ${ }^{a}$ School of Aerospace Engineering, Beijing Institute of Technology, Beijing 100081, China \\ ${ }^{\mathrm{b}}$ State Key Laboratory of High Temperature Gas Dynamics, Institute of Mechanic, Chinese Academy of Sciences, Beijing 100190, China \\ ${ }^{\mathrm{c}}$ School of Engineering Sciences, University of Chinese Academy of Sciences, Beijing 100049, China
}

\section{A R T I C L E I N F O}

\section{Keywords:}

Oblique detonation

Hydrogen

Initiation mechanism

Critical

\begin{abstract}
A B S T R A C T
Two-dimensional, oblique detonation waves (ODWs) in a stoichiometric hydrogen-air mixture are simulated using the reactive Euler equations with a detailed chemical reaction model. This study focuses the effects of expansion waves on the initiation, which is modeled by a finite-length wedge. Numerical results demonstrate that the expansion wave may quench the ODW if it interacts with the initiation region, and the critical position is found to be dependent on the incident Mach number $M_{0}$. The critical position moves upstream in the case of high $M_{0}$, and downstream in the case of low $M_{0}$. Furthermore, ODW structures show different behaviors when the expansion wave is near its critical position. In the case of $M_{0}=10$, the structure is featured by a stationary but decoupled shock and reactive surface, while a transient downstream-moving ODW is observed in the case of $M_{0}=7$. By decreasing the turning angle, the former one keeps the same, while the later one becomes also stationary. These differences are related with the initiation mechanisms of two ODW structures, demonstrating that the structure of wave-controlled initiation is more sensitive to the expansion waves than the kinetic-controlled initiation.
\end{abstract}

\section{Introduction}

Detonations are supersonic combustion waves travelling in premixed combustible mixtures, in which the strong leading shock and heat release are closely coupled [1,2]. The detonation research is useful and crucial for automotive internal combustion engines to prevent the super-knock phenomena [3,4]. More importantly, detonation engines used in the future aircrafts attract more and more attention due to the high thermal cycle efficiency. There are three kinds of detonation engines, which are pulse detonation engines [5], rotating detonation engines [6,7] and oblique detonation engines [8], respectively. Oblique detonation engines are one kind of ramjet engines in which oblique detonation wave (ODW) is triggered to achieve the fast and high efficient combustion. It has the potential to be used in the hypersonic propulsion, operating at the higher flight Mach number than Scramjet (supersonic combustion ramjet). In order to develop the practical ODWbased engines, it is necessary to get more fundamental understanding of the oblique detonation structure and instability.

ODW structures were usually simplified to be the oblique shock waves and post-shock release zones [9] initially, but further studies [10-14] demonstrate that the surface of ODW is composed of a nonreactive oblique shock before the oblique detonation surface forms. There are several studies on surface instability of ODW recently [15-20], illustrating and quantifying the formation of fine-scale structures on oblique detonation surfaces. For simplicity, most of the previous numerical studies [10-20] used simplified chemical reaction models, mainly the one-step irreversible Arrhenius kinetic model, which has interior flaws in the initiation study. Recent studies [21-24] demonstrate that the complicated chemistry has obvious effects on the detonation characteristics, so ODW studies based on detailed chemistry model are necessary to be performed. Current studies mainly use the hydrogen-air mixtures, whose detailed chemical mechanisms are widely studied for tens of years. ODW structures influenced by the inflow Mach numbers and gas-dynamic parameters, such as inflow pressure and temperature, are simulated and discussed [25]. To study the ODW influenced by the fuel injection, the inflow inhomogeneity is modeled and simulated, demonstrating the distorted reaction surfaces and morphology variation of the ODW structures [26,27]. Our recent study demonstrated that there are two initiation mechanisms of ODW in the wedge-induced structures in hydrogen-air mixtures [28]. In the case of high incident Mach number, the temperature behind the oblique shock is high enough to achieve the self-ignite, so it is called the

\footnotetext{
* Corresponding author.

E-mail address: hhteng@bit.edu.cn (H. Teng).
} 
kinetics-controlled mechanism. On the other side, in the case of low incident Mach number, the temperature is not high enough so that the initiation position is determined by the interaction of the oblique shock and oblique detonation, which is called wave-controlled mechanism. The initiation length of kinetics-controlled ODW can be calculated from the constant-volume combustion theory, but the initiation length of wave-controlled ODW is still lack of theoretical model, which requires more knowledge on the wave dynamics of ODW structures.

To deepen our understanding on the ODW initiation, this study simulated the ODW using a finite-length wedge, replacing the semi-infinite wedge in the previous study [28]. Due to the effects of expansion waves, the initiation features of ODW structures may change and induce complicated phenomena. In the detonation instability and structure evolution [29-31], effects of expansion waves have been simulated and discussed. Papalexandris [32] found out that the expansion wave may quench the ODW, but it is still lack of the detailed study on the effects of expansion wave on ODW initiation. In this study, the ODW structures influenced by the expansion waves are simulated. By analyzing two typical cases, the interaction of expansion wave and ODW are studied to further our understanding on the ODW initiation.

\section{Physical and mathematical models}

A schematic of the oblique detonation wave induced by the wedge in a combustible gas mixture is shown in Fig. 1. The wedge in supersonic inflow induces an oblique shock wave (OSW) first. If the inflow is combustible mixtures with high incident Mach number $M_{0}$, an exothermic chemical reaction may begin and result in oblique detonation. The computational domain is shown in region enclosed by the dashed line, and the Cartesian grid is aligned with the wedge surface. Similar to previous numerical studies on oblique detonations [28], the present analysis is based on the two-dimensional multi-species Euler equations written as follows:

$\frac{\partial \widetilde{U}}{\partial t}+\frac{\partial \widetilde{F}}{\partial \xi}+\frac{\partial \widetilde{G}}{\partial \eta}=\widetilde{S}$

where

$\widetilde{U}=\frac{U}{J}, \widetilde{F}=\frac{1}{J}\left(\xi_{x} F+\xi_{y} G\right), \widetilde{G}=\frac{1}{J}\left(\eta_{x} F+\eta_{y} G\right), \widetilde{S}=\frac{S}{J}$

with

$U=\left\{\begin{array}{c}\rho_{1} \\ \vdots \\ \rho_{n} \\ \rho u \\ \rho v \\ e\end{array}\right\}, \quad F=\left\{\begin{array}{c}\rho_{1} u \\ \vdots \\ \rho_{n} u \\ \rho u^{2}+p \\ \rho u v \\ (e+p) u\end{array}\right\}, G=\left\{\begin{array}{c}\rho_{1} v \\ \vdots \\ \rho_{n} v \\ \rho u v \\ \rho v^{2}+p \\ (e+p) v\end{array}\right\}, \quad S=\left\{\begin{array}{c}\omega_{1} \\ \vdots \\ \omega_{n} \\ 0 \\ 0 \\ 0\end{array}\right\}$

and

$\frac{1}{J}=\frac{\partial(\xi, \eta)}{\partial(x, y)}=\left|\begin{array}{ll}\xi_{x} & \xi_{y} \\ \eta_{x} & \eta_{y}\end{array}\right|=\xi_{x} \eta_{y}-\xi_{y} \eta_{x}$

In the above equations, the total density and total energy are

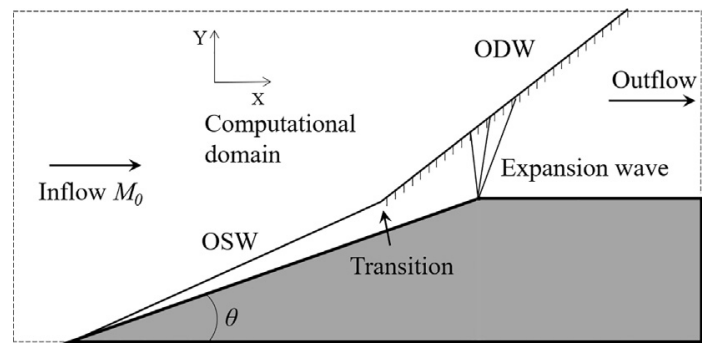

Fig. 1. Sketch of the oblique detonation simulation. calculated by

$\rho=\sum_{i=1}^{n} \rho_{i}, \quad e=\rho h-p+\frac{1}{2} \rho\left(u^{2}+v^{2}\right)$

where specific enthalpy can be written as $h=\sum_{i=1}^{n} \rho_{i} h_{i} / \rho$ with $h_{i}$ obtained from thermodynamic data of each individual specie. The equation of state is

$p=\sum_{i=1}^{n} \rho_{i} \frac{R_{0}}{w_{i}} T$

where $w_{i}$ is the molecular weight and $T$ is the gas temperature; $\omega_{i}$ is the species' specific mass production rate, which is dictated by the chemical reaction model.

The governing equations are discretized on Cartesian grids and solved with the DCD scheme [33] with Strang's splitting, and the algorithm has been used to study the complicated flows [34,35]. The lastest $\mathrm{H}_{2} / \mathrm{O}_{2}$ kinetic model for high-pressure combustion [36-38] is used here, which involves 27 reversible elementary reactions among the 8 species $\left(\mathrm{H}_{2}, \mathrm{O}_{2}, \mathrm{H}_{2} \mathrm{O}, \mathrm{H}, \mathrm{O}, \mathrm{OH}, \mathrm{HO}_{2}\right.$, and $\left.\mathrm{H}_{2} \mathrm{O}_{2}\right)$ with 5 nonreacting species $\left(\mathrm{N}_{2}, \mathrm{Ar}, \mathrm{He}, \mathrm{CO}\right.$, and $\left.\mathrm{CO}_{2}\right)$. Thermodynamic properties of the chemical species are evaluated from the 9-coefficient NASA polynomial representation [39]. A stoichiometric hydrogen-air mixture with $\mathrm{H}_{2}: \mathrm{O}_{2}: \mathrm{N}_{2}=2: 1: 3.76$ is used, with the temperature $300 \mathrm{~K}$ and the pressure $101,325 \mathrm{~Pa}$. The uniform flow is set as the initial condition, whose velocities are calculated from the incident Mach number $M_{0}$. The slip reflecting boundary condition is used on the wedge surface and the other boundaries are interpolated under the assumption of zero firstorder derivatives of all flow parameters. Since the characteristic lengths of chemical reactions vary in a wide range, both the computational domain and mesh scale are adjusted. For each case, the results are examined to assure the mesh scale effects are trivial enough. Unless specified, a wedge angle $\theta$ is fixed to be $25^{\circ}$ and the turning angle inducing the expansion waves is also $25^{\circ}$, so the outflow is paralleled with the inflow.

\section{Numerical results and discussion}

\subsection{ODW influenced by the expansion waves}

The flow fields of ODW structures with $M_{0}=10$ and 7 are shown in Fig. 2, with the upper displaying results from low resolution and the lower from high resolution. In the low-resolution simulations, the grid of the left boundary is $0.005 \mathrm{~mm} * 0.005 \mathrm{~mm}$ in the case of $M_{0}=10$, and $0.05 \mathrm{~mm} * 0.05 \mathrm{~mm}$ in the case of $M_{0}=7$. The grid $500 * 400$ is employed in both cases, so the grid scale around the initiation points becomes about $0.005 \mathrm{~mm} * 0.003 \mathrm{~mm}$ in the case of $M_{0}=10$ and $0.05 \mathrm{~mm} * 0.03 \mathrm{~mm}$ in the case of $M_{0}=7$, respectively. Obvious differences can be observed between the flow fields shown in Fig. 2a and b. The smooth transition appears in Fig. 2a, while the abrupt transition appears in Fig. 2b, which is physically reasonable according to our previous study [13]. Because of the difference of incident Mach number $M_{0}$, the initiation length of inert shock in the case of $M_{0}=7$ is almost 10 times of that in the case of $M_{0}=10$.

Resolution tests are performed and the results are compared in both frames in Fig. 2. The computational domain of the case $M_{0}=10$ is $2.5 \mathrm{~mm} * 2.0 \mathrm{~mm}$, while the domain of the case $M_{0}=7$ is $25 \mathrm{~mm} * 20 \mathrm{~mm}$. In the both cases, the high-resolution simulation uses the grid $1000 * 800$, doubling the grid numbers in both directions. Obviously, the general ODW structures of two cases are similar to each other, although some slight differences can be observed. Low resolution induces the initiation moving downstream a little in Fig. 2a, while moving upstream a little in Fig. 2b. However, it should be noted that the low resolution is not adequate to resolve all the chemical kinetic scales, such as the behaviors on the slip line instability observed shown in Fig. 2b. Despite of the observed differences, for the purpose of this 

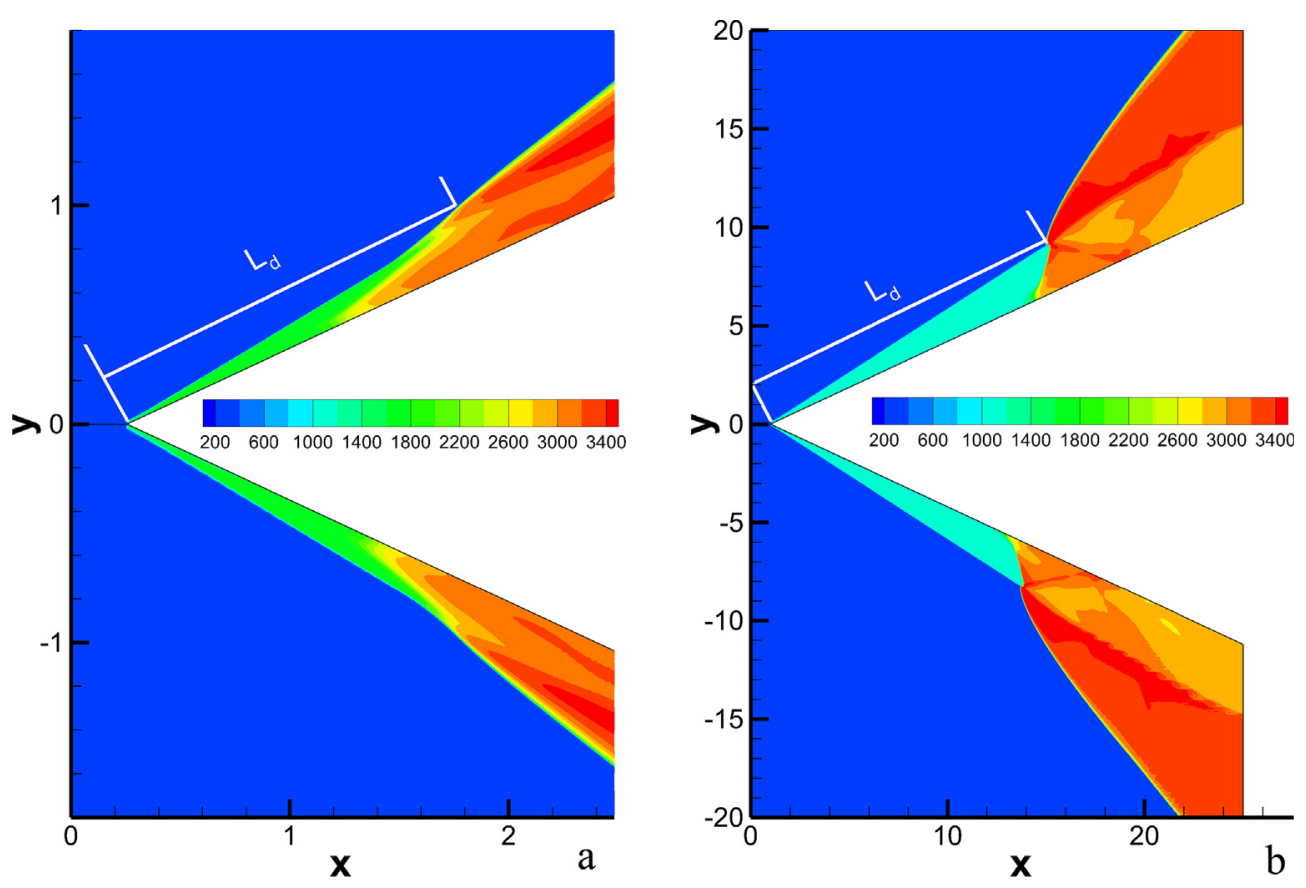

Fig. 2. Temperature of ODW structures with $M_{0}=10$ (a) and 7 (b).

work, the chosen grid size provides converged, global, formation structures, sufficient to guarantee the reliability of the conclusion.

Based on the above results reproduced by our previous study [28], we introduce the turning angle to the further simulations to study the effects of expansion waves. As pointed out by previous studies [29,32], the expansion waves may quench the ODW if they locate upstream enough. However, aforementioned results demonstrate that the length scales of two cases are different, and the initiation length in the case of $M_{0}=7$ is almost 10 times of that in the case of $M_{0}=10$. To perform the quantity analysis, we define $L_{e}$ to denote the position of expansion waves, and select $L_{d}$ to nondimensionalize $L_{e}$, which makes the results from different cases can be compared together. $L_{e}$ denotes the length from the wedge tip to the location of expansion wave, while $L_{d}$ denotes the length of the initiation region along the wedge in the case without considering the expansion waves. In the abrupt transition, the end of $L_{d}$ is multi-wave point projection on the wedge. In the smooth transition, there is an inflexion point on the curved shock, and we use its projection on the wedge.

With $L_{e} / L_{d}=1.0$ and 0.8 , the flow fields of $M_{0}=10$ and 7 are shown in Figs. 3 and 4, respectively. Generally, the ODW initiation and its position are not influenced in the cases of $L_{e} / L_{d}=1.0$, although the expansion waves are introduced and the downstream flow fields are changed. Decreasing $L_{e} / L_{d}$ to be 0.8 , the ODW initiates successfully when $M_{0}=10$, while quenches when $M_{0}=7$. Further decreasing $L_{e} / L_{d}$ to be 0.6 , both ODW cannot be initiated, and the results are similar to those in the unreactive gas, not shown here. The ODW quench induced by the expansion waves has been observed before, but this study demonstrates that the effects are also dependent on the ODW structures, never been proposed before and needs to be studied deeply.

\subsection{Critical structures dependent on the expansion location}

To investigate the reason of different expansion effects, the parameter $L_{e} / L_{d}$ is adjusted to get the critical cases. As shown in Fig. 3, the wave structures are similar in the cases of $L_{e} / L_{d}=1.0$ and 0.8 , and cannot be initiated in the case of $L_{e} / L_{d}=0.6$. By adjusting $L_{e} / L_{d}$ to be 0.65, the pressure and temperature are shown in Fig. 5, illustrating a special structure between the successful initiation and fully quench. In usual ODWs, the pressure will increase and the oblique shock angle rises resulting from the heat release, which are both absent in this structure. However, the combustion effect can be observed in Fig. 5b, demonstrating a reactive surface almost paralleled with the oblique shock. The heat release should be triggered by the oblique shock, but it is suppressed by the expansion waves, so does not result in the detonation initiation.

Fig. 6 shows pressure and temperature curves along the lines $y=0.5$ and 0.8 of the flow fields shown in Fig. 5. On the line $y=0.5$, the pressure rising derived from the heat release can be observed
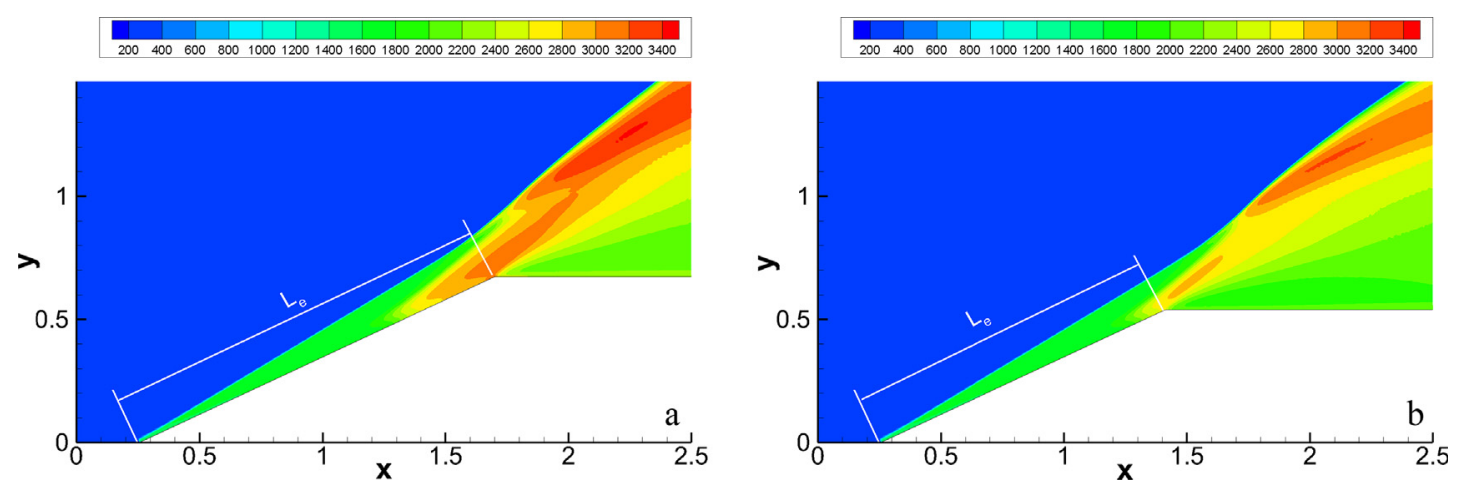

Fig. 3. Temperature of ODW structures with $M_{0}=10, L_{e} / L_{d}=1.0$ (a) and 0.8 (b). 

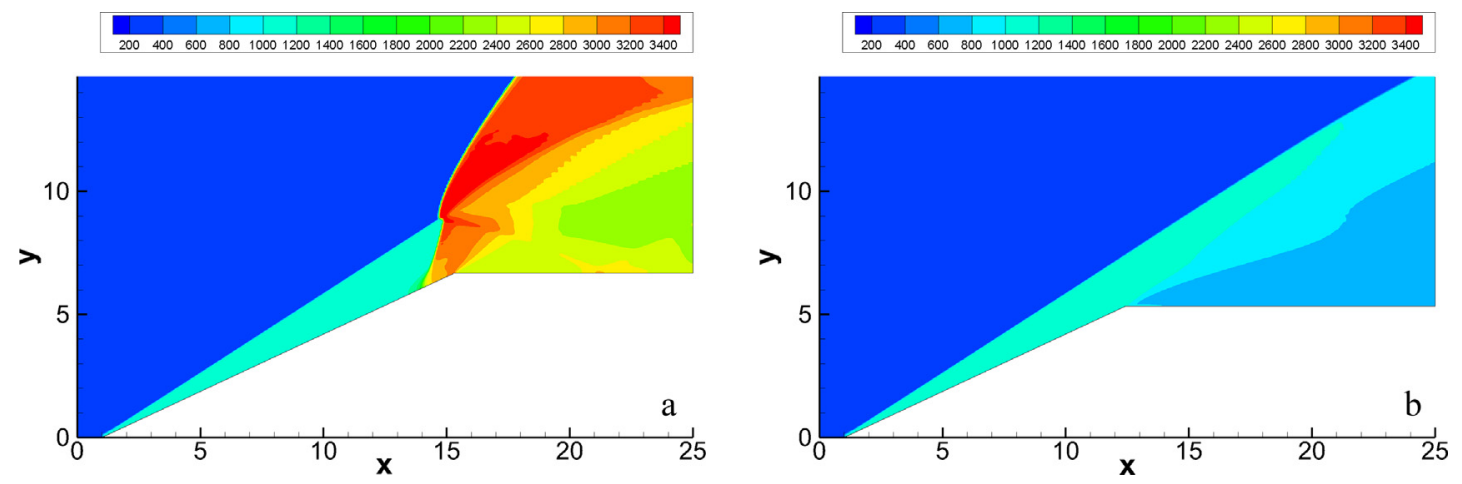

Fig. 4. Temperature of ODW structures with $M_{0}=7, L_{e} / L_{d}=1.0$ (a) and 0.8 (b).
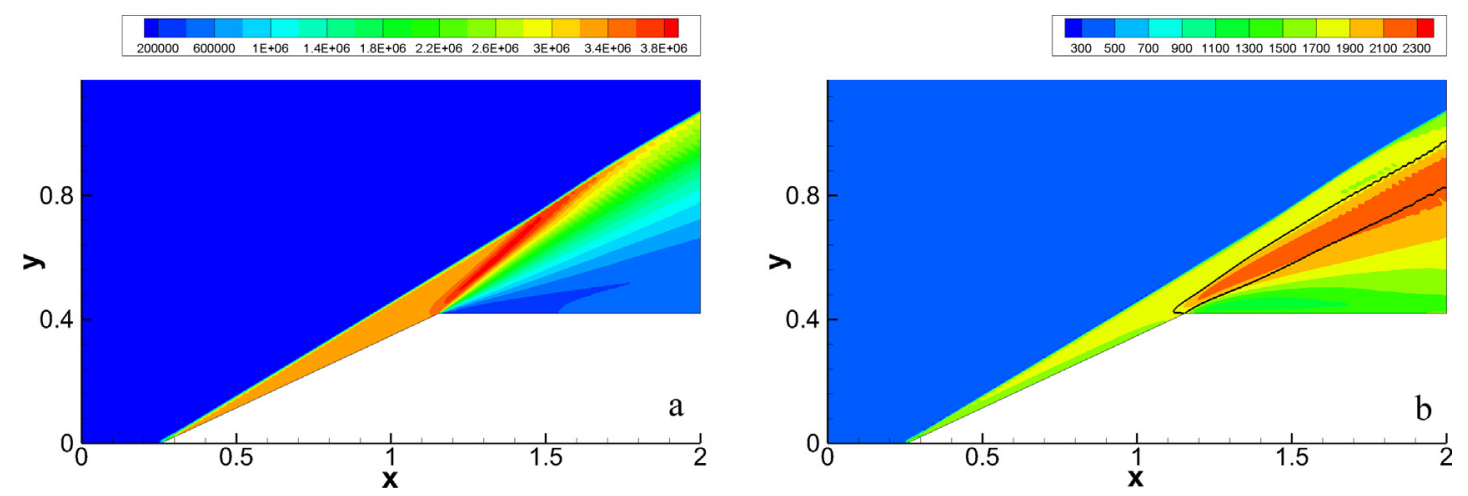

Fig. 5. Pressure (a) and temperature (b) of ODW structures with $M_{0}=10$ and $L_{e} / L_{d}=0.65$.

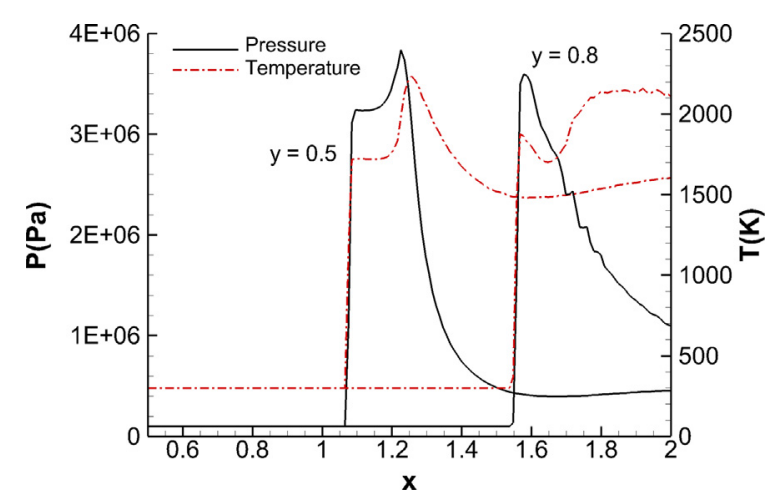

Fig. 6. Pressure and temperature along the lines $y=0.5$ and 0.8 .

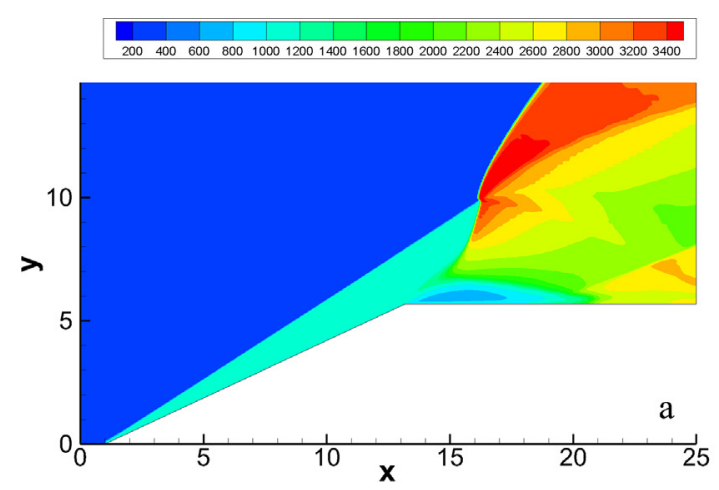

Fig. 7. Evolution of the ODW structure with $M_{0}=7$ and $L_{e} / L_{d}=0.85$ (a) initial stage; (b) after long time iteration.

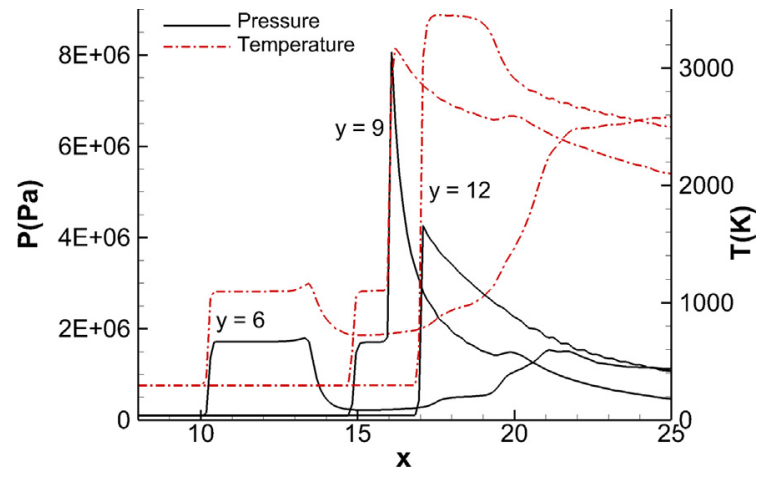

Fig. 8. Pressure and temperature along the lines $y=6,9$ and 12 with $M_{0}=7$ and $L_{e} / L_{d}=0.85$. 

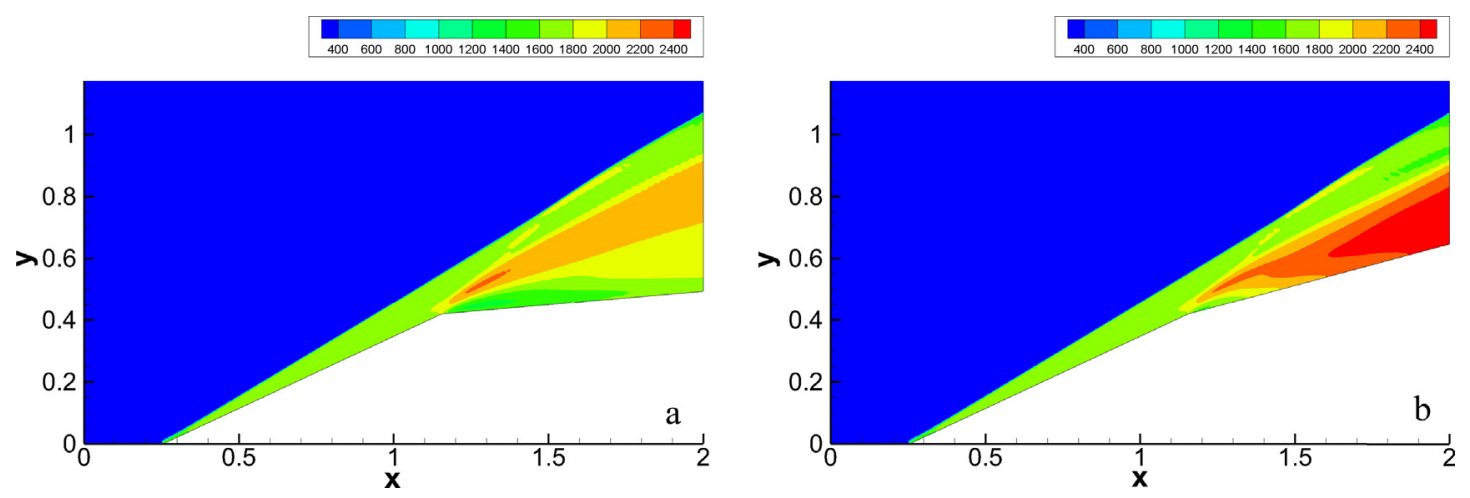

Fig. 9. Temperature of ODW structures with $M_{0}=10$ and $L_{e} / L_{d}=0.65$, turning angle $20^{\circ}$ (a) and $10^{\circ}$.
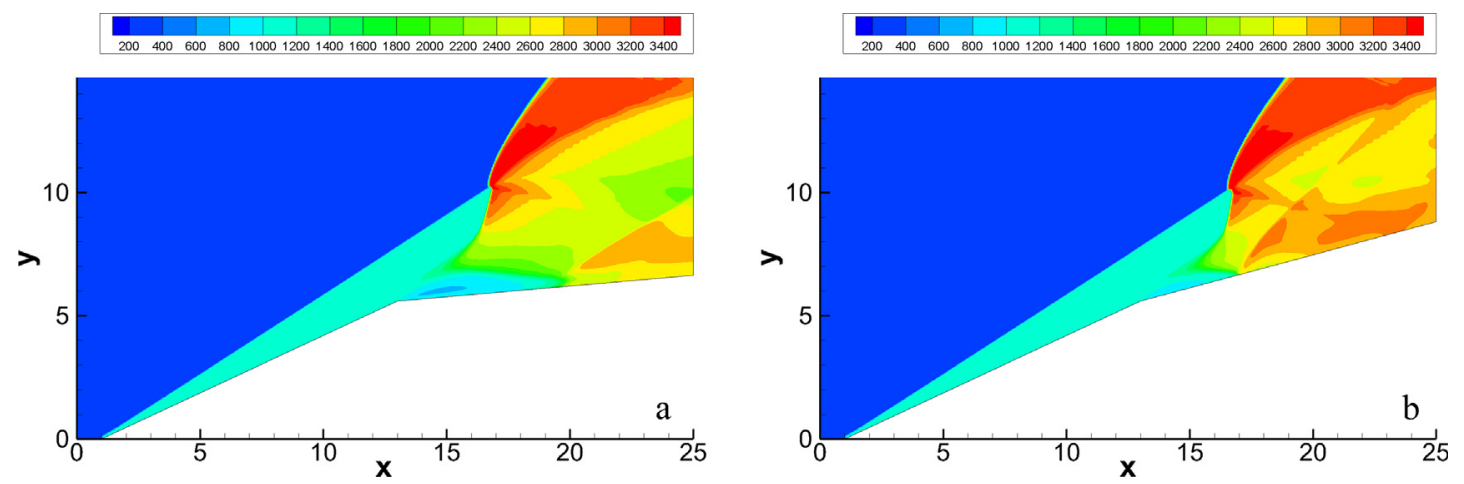

Fig. 10. Temperature of ODW structures with $M_{0}=7$ and $L_{e} / L_{d}=0.85$, turning angle $20^{\circ}$ (a) and $10^{\circ}$.

clearly, denoting by the coupled pressure and temperature peak. However, the pressure and temperature decrease subsequently, which should attribute to the effects of the expansion waves. On the line $y=0.8$, the expansion effects dominate the post-shock flow, so the pressure peak is induced by the oblique shock and a decoupled reactive surface forms. Generally, an intermediate structure between the successful initiation and fully quench is observed, which should be attributed into the competition of the heat release and expansion effects.

In the case of $M_{0}=7$ and $L_{e} / L_{d}=0.8$, ODW can't be initiated as shown in Fig. 4, so $L_{e} / L_{d}$ is increased and the resulting flow fields with $L_{e} / L_{d}=0.85$ is shown in Fig. 7. The ODW is initiated first as shown in Fig. 7a, whose structure is similar to that with $L_{e} / L_{d}=1.0$ in Fig. 4a. Nevertheless, this structure cannot sustain for a long time. After the quick initiation, this structure moves downstream slowly. As shown in Fig. 7b, the structure will finally move outside the computational domain, so the structure is featured by a transient downstream-moving ODW. Fig. 8 shows the pressure and temperature along the lines $y=6$, 9 and 12 of the flow fields in Fig. 7a, illustrating the parameters of three typical positions. Due to the pressure and temperature decrease, the expansion effects can be observed clearly on the line $y=6$. However, the detonations are initiated along the lines $y=9$ and 12 , so the expansion effects are hard to be distinguished. Because the gas along $y=9$ is compressed by the oblique shock, the peak pressure is higher than that along $y=12$.

To clarify the effects of expansion wave, more cases with different $M_{0}$, i.e. 8 and 9 , are simulated. Numerical results demonstrate that ODW is initiated successfully with $L_{e} / L_{d}=0.85$ in the cases of $M_{0}=8$ and 9. In the case of $M_{0}=8$, decreasing $L_{e} / L_{d}$ to be 0.75 results into a structure like that in Fig. 7a, i.e. successful initiation, but it persists and does not move downstream, like the case with $M_{0}=10$. Further decreasing $L_{e} / L_{d}$ may quench the ODW. In the case of $M_{0}=9$, similar phenomena are observed, but the critical $L_{e} / L_{d}$ decrease to be 0.70 . Generally, the critical $L_{e} / L_{d}$ decreases when $M_{0}$ increases, and the ODW behaviors of the cases $M_{0}=8$ and 9 can be viewed as the intermediate situations of two extreme cases of $M_{0}=7$ and 10 .

\subsection{Dependence of expansion effects on the turning angle}

Aforementioned results demonstrate that the expansion effects are different when the initiation mechanisms are different. To further elucidate these two different critical features, additional cases with different turning angles are introduced. Different from above cases with the same turning angle as the wedge angle, i.e. $25^{\circ}$, the following cases change the turning angle to be $20^{\circ}$ and $10^{\circ}$. Decreasing the turning angles actually weakens the expansion effects, and the resulting structures are shown in Figs. 9 and 10. In the case $M_{0}=10$, the general structures keep the same, featured by the intermediate structure. Conversely, in the case of $M_{0}=7$, the wave structure will not move downstream, and the stationary flow fields are shown in Fig. 10. Therefore, the change of turning angle plays more important role in the low $M_{0}$ cases.

As pointed out by our previous study [20], the initiation is kineticscontrolled in the case of $M_{0}=10$, while wave-controlled in the case of $M_{0}=7$. The initiation of kinetics-controlled ODW is actually a self-ignition process, so the interaction between different streamlines is weak. The weak interaction leads to the structure which is not sensitive to the expansion waves, so the critical structures appear at around $L_{e} /$ $L_{d}=0.65$, and the change of turning angle does not change the critical structure. However, in the wave-controlled cases, there is strong interaction of different streamlines, so the structure is sensitive to the expansion waves. Not only $L_{e} / L_{d}$ corresponding critical structures move from 0.65 to 0.85 , but also the behavior of the critical structures changes. Therefore, these differences are related with the initiation mechanisms of two ODW structures, demonstrating that the structure of wave-controlled initiation is more sensitive to the expansion waves than the kinetic-controlled initiation. 


\section{Conclusion}

Oblique detonations induced by a finite wedge in a stoichiometric hydrogen-air mixture are simulated, and this study focuses on the effects of expansion waves which are induced by the turning angle due to the finite wedge. Based on previous studies, two typical initiation structures, which are controlled by $M_{0}$, are chosen to be the basic cases. Numerical results demonstrate that the expansion effects are dependent on the structures, i.e. $M_{0}$. The nondimensional length $L_{e} / L_{d}$ is introduced to quantify the expansion wave location, and numerical results demonstrate that its critical value becomes large when $M_{0}$ decreasing. Furthermore, the ODW structures show very different features at the critical cases. The former one is a stationary but decoupled shock and reactive surface, and the latter one is a transient downstreammoving ODW. Additional cases with $M_{0}=8$ and 9 show the intermediate situations of two extreme cases of $M_{0}=7$ and 10. By decreasing the turning angle, the former one keeps the same, while the later one becomes also stationary. These different effects of expansion waves derive from the different initiation mechanisms dependent on $M_{0}$, which is important in both the fundamental and engineering studies.

\section{Acknowledgment}

The research is supported by NSFC No. 91641130 and 11372333.

\section{References}

[1] Fickett W, Davis WC. Detonation: theory and experiment. Dover Publications, Courier Corporation; 2000.

[2] Lee JHS. The detonation phenomenon. New York: Cambridge University Press; 2008.

[3] Wang Z, Qi Y, He X, et al. Analysis of pre-ignition to super-knock: hotspot-induced deflagration to detonation. Fuel 2015;144:222-7.

[4] Wang Z, Liu H, Reitz RD. Knocking combustion in spark-ignition engines. Prog Energy Combust Sci 2017;61:78-112.

[5] Roy GD, Frolov SM, Borisov AA, Netzer DW. Pulse detonation propulsion: challenges, current status, and future perspective. Prog Energy Combust Sci 2004;30:545-672.

[6] Wolanski P. Detonative propulsion. Proc Combust Inst 2013;34:125-58.

[7] Braun EM, Lu FK, Wilson DR, Camberos JA. Airbreathing rotating detonation wave engine cycle analysis. Aerosp Sci Technol 2013;27:201-8.

[8] Lu FK, Fan H, Wilson DR. Detonation waves induced by a confined wedge. Aerosp Sci Technol 2006;10(8):679-85.

[9] Pratt DT, Humphrey JW, Glenn DE. Morphology of standing oblique detonation waves. J Propul Power 1991;7(5):837-45.

[10] Li C, Kailasanath K, Oran ES. Detonation structures behind oblique shocks. Phys Fluids 1994;4:1600-11.

[11] Viguier C, Figueira da Silva LF, Desbordes D, Deshaies B. Onset of oblique detonation waves: comparison between experimental and numerical results for hydrogen-air mixtures. In: Symposium (International) on Combustion, vol. 26(2); 1996. p. 3023-31.

[12] Figueira da Silva LF, Deshaies B. Stabilization of an oblique detonation wave by a wedge: a parametric numerical study. Combust Flame 2000;121:152-66.

[13] Teng HH, Jiang ZL. On the transition pattern of the oblique detonation structure. $J$ Fluid Mech 2012;713:659-69.

[14] Wang T, Zhang Y, Teng H, Jiang Z, Ng HD. Numerical study of oblique detonation wave initiation in a stoichiometric hydrogen-air mixtur. Phys Fluids 2015;27(9):096101.

[15] Choi JY, Kim DW, Jeung IS, Ma F, Yang V. Cell-like structure of unstable oblique detonation wave from high-resolution numerical simulation. Proc Combust Inst 2007;31:2473-80.

[16] Teng HH, Jiang ZL, Ng HD. Numerical study on unstable surfaces of oblique detonations. J Fluid Mech 2014;744:111-28.

[17] Gui MY, Fan BC, Dong G. Periodic oscillation and fine structure of wedge-induced oblique detonation waves. Acta Mech Sin 2011;27:922-8.

[18] Verreault J, Higgins AJ, Stowe RA. Formation of transverse waves in oblique detonations. Proc Combust Inst 2013;34(2):1913-20.

[19] Teng H, Ng HD, Li K, Luo C, Jiang Z. Cellular structure evolution on oblique detonation surfaces. Combust Flame 2015;162:470-7.

[20] Yang P, Ng HD, Teng H, Jiang Z. Initiation structure of oblique detonation waves behind conical shocks. Phys Fluids 2017;29(8):086104.

[21] Zhang B, Bai CH. Methods to predict the critical energy of direct detonation initiation in gaseous hydrocarbon fuels - an overview. Fuel 2014;117:294-308.

[22] Zhang B, Shen XB, Pang L, Gao Y. Methane-oxygen detonation characteristics near their propagation limits in ducts. Fuel 2016;177:1-7.

[23] Zhang B. The influence of wall roughness on detonation limits in hydrogen-oxygen mixture. Combust Flame 2016;169:333-9.

[24] Zhang B, Shen XB, Pang L, Gao Y. Measurement and prediction of detonation cell size in binary fuel blends of methane/hydrogen mixtures. Fuel 2016;172:196-9.

[25] Teng H, Zhang Y, Jiang Z. Numerical investigation on the induction zone structure of the oblique detonation waves. Comput Fluids 2014;95:127-31.

[26] Fang Y, Hu Z, Teng H, Jiang Z, Ng HD. Effects of inflow equivalence ratio inhomogeneity on oblique detonation initiation in hydrogen-air mixtures. Aerosp Sci Technol 2017;71:256-63.

[27] Iwata K, Nakaya S, Tsue M. Wedge-stabilized oblique detonation in an inhomogeneous hydrogen-air mixture. Proc Combust Inst 2017;36(2):2761-9.

[28] Teng H, Ng HD, Jiang Z. Initiation characteristics of wedge-induced oblique detonation wave in a stoichiometric hydrogen-air mixture. Proc Combust Inst 2017;36(2):2735-42.

[29] Choi JY, Shin EJ, Jeung IS. Unstable combustion induced by oblique shock waves at the non-attaching condition of the oblique detonation wave. Proc Combust Inst 2009;32(2):2387-96.

[30] Bhattrai B, Tang H. Formation of near-Chapman-Jouguet oblique detonation wave over a dual-angle ramp. Aerosp Sci Technol 2017;63:1-8.

[31] Liu Y, Han X, Yao X, Wang J. A numerical investigation of the prompt oblique detonation wave sustained by a finite-length wedge. Shock Waves 2016;26(6):729-39.

[32] Papalexandris MV. A numerical study of wedge-induced detonations. Combust Flame 2000;120(4):526-38.

[33] Jiang ZL. On dispersion-controlled principles for non-oscillatory shock-capturing schemes. Acta Mech Sin 2004;20(1):1-15.

[34] Hu ZM, Wang C, Jiang ZL, Khoo BC. On the numerical technique for the simulation of hypervelocity test flows. Comput Fluids 2015;106:12-8.

[35] Hu ZM, Dou SH, Khoo BC. On the modified dispersion-controlled dissipative (DCD) scheme for computation of flow supercavitation. Comput Fluids 2011;40:315-23.

[36] Burke MP, Chaos M, Ju Y, Dryer FL, Klippenstein SL. Comprehensive H2/O2 kinetic model for high-pressure combustion. Int J Chem Kinet 2012;44(7):444-74.

[37] Li J, Zhao Z, Kazakov A, Dryer FL. An updated comprehensive kinetic model of hydrogen combustion. Int J Chem Kinet 2004;36:566-75.

[38] Taylor BD, Kessle DA, Gamezo VN, Oran ES. Numerical simulations of hydrogen detonations with detailed chemical kinetics. Proc Combust Inst 2013;34:2009-16.

[39] Bonnie JM, Michael JZ, Gordon S. NASA Glenn Coefficients for Calculating Thermodynamic Properties of Individual Species. NASA/TP, 2002-211556. 